\title{
Evolution Subject to an Energy Driven Fundamental Time Arrow
}

\author{
Helmut Tributsch \\ Bio-Mimetics in Energy Systems Program, Carinthia University for Applied Sciences, Villach, Austria \\ Email: helmut.tributsch@alice.it
}

How to cite this paper: Tributsch, H. (2018). Evolution Subject to an Energy Driven Fundamental Time Arrow. Advances in Anthropology, 8, 140-174.

https://doi.org/10.4236/aa.2018.83008

Received: July 30, 2018

Accepted: August 25, 2018

Published: August 28, 2018

Copyright (C) 2018 by author and Scientific Research Publishing Inc. This work is licensed under the Creative Commons Attribution International License (CC BY 4.0).

http://creativecommons.org/licenses/by/4.0/

\begin{abstract}
Adopting a fundamental time arrow in nature, a free energy aiming at decreasing its presence per state by generating a flow of action, as recently derived from the principle of least action, entrains significant consequences for understanding mechanisms in evolution and biology. Simultaneously, the presently favoured statistical, entropic time arrow is tolerated as a limiting case. The entropy law, which self-organized systems follow, is deducible and time reading for feedback processes is functioning at very short time scale during energy turnover, even during build-up of order (decrease of entropy). As a consequence, living systems can self-organize on molecular level and evolution has an aim. This aim is maximizing entropy and thus energy turnover, within given constraints. Genetic mechanisms, here seen as self-organized chemical information handling, had to deal with such a parallel entropy (energy) aim of self-organized living structures and should be considered superposed, adapted to it and controlling it. Since increasing energy turnover is paralleled by an increasing ability of self-organized systems for generating order, this explains evolution towards higher perfection and the trend towards symbiotic energy harvesting. Information as well, which has an energy content, can self-organize within the neuronal structure of the brain and create a higher hierarchy of information handling. It is recognized as consciousness and spirit, which thus can be explained on purely materialistic basis. Evolution towards maximum energy (entropy) turnover as a fate for human civilization, when restraints are further decreasing, is a pessimistic prospect due to unavoidable environmental consequences. However, evolution of spirit is a straightforward parallel consequence and this may be considered the ultimate aim of evolution. The introduction of a fundamental energy driven time arrow as a paradigm change is verifiable via convincing falsification conditions and would revolutionize our understanding of life and human evolution within a significantly more friendly universe.
\end{abstract}




\section{Keywords}

Time Arrow, Self-Organization, Maximum Entropy Production, Aim of Evolution, Consciousness, Spirit

\section{Introduction}

\subsection{Time Is Now Considered an Illusion, Just Used as an Ordering Parameter}

Science has an ambivalent relation to time. On one hand, the experience is that everything is moving in one direction only, including evolution. On the other hand, physics deals with time neutral particles, considers time to be an illusion (a statement, for example, by A. Einstein in a letter to the family of his passed away friend Besso) and carefully avoids expressing time in fundamental laws and formulas. They are all formulated in a way that allows them to function both in positive and negative time direction. The only time direction, which science is presently admitting, because of empirical evidence, is the statistical, probabilistic one, which is showing the obvious direction of increasing disorder, entropy. It is, in fact not a real time arrow, because it is not pushing time, the progress of changes, as a time arrow should do. Statistical, probabilistic processes just appear to favour this time direction (and not the opposite one). For reasons of simplicity, it will nevertheless be called "statistical" or "probabilistic" time arrow.

The mathematical derivation and implementation of this statistical time direction, or statistical time arrow, starting with Boltzmann's H-theorem (intended to describe entropy increase), is, strictly speaking, based on a questionable mathematical procedure: the time invertible properties of a large number of time neutral gas particles are mathematically described, but then drastic simplifications are introduced by grouping particles with comparable properties together for further simplified calculations. All together information on the particles is thrown away. And today we know that information has an energy content. Energy was consequently thrown out of the system to demonstrate that there is no way back for the studied, evolving system to the start. Such a result is, on the basis of thrown away information, to be expected, since the system is not any more the original one. This questions the mathematical concept of a statistical time arrow, which anyhow cannot explain why probabilistic time does not proceed into the past, which would be equally derivable with the same mathematical procedure (Loschmidt paradox). The statistical entropic time arrow relies on the probable behaviour of a large number of particles, which cannot find back to the original order. Figure 1(a) shows this in a simplified way via a box, in which gas particles are first concentrated in one part of the box before spreading into the entire box, approaching a condition of maximum probability or disorder corresponding to maximum entropy. This behaviour corresponds to the empirical statement of the second law of "equilibrium" thermodynamics with respect to 


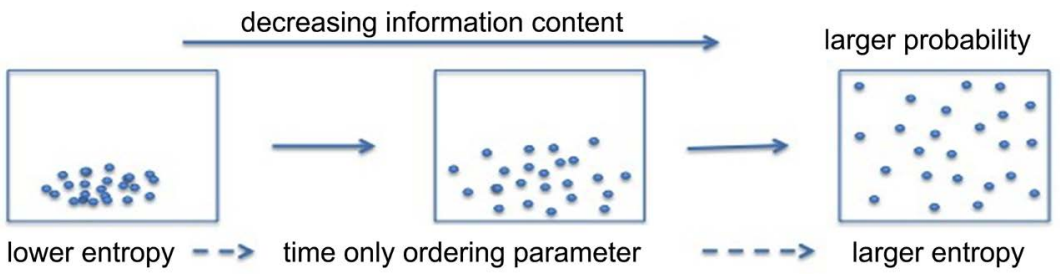

(a)

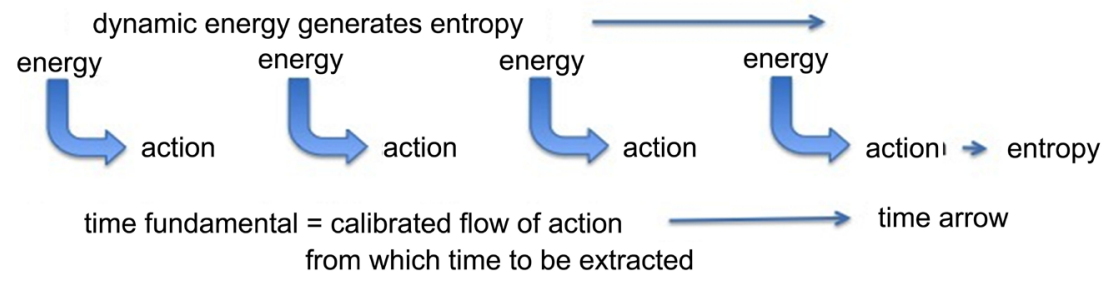

(b)

Figure 1. The statistical, probabilistic time arrow in direction of increasing entropy (a) is compared with the proposed fundamental time arrow (b), where not probability but a dynamic energy is driving time (Tributsch, 2016a) (further explanation in the text).

entropy. It is not obvious how such a statistical time arrow could support feedback processes for self-organization. Is such a purely statistical, probabilistic time arrow, together with an energy without any "dynamic" property towards producing action, adequate for a description of molecular biological processes and of evolution, where molecular structures exposed to feedback processes have to react in a time oriented way towards an increase of order and a decrease of probability (decrease of entropy)? In addition, present science cannot yet give a statement on the expected entropy (energy) behaviour of such self-organized systems, which are subject to non-linear irreversible behaviour.

\subsection{Why Is a Fundamental Energy Driven Time Arrow Crucial for Biology?}

Life and its most important mechanisms are self-organized processes. It is known that they function far from thermodynamic equilibrium and involve feedback processes (compare scheme in Figure 2). Without feedback processes, that distinguish between "before" and "after" dynamic self-organization would simply not be possible. This can mathematically clearly be demonstrated, since without feedback processes the necessary complexity and order could not be achieved.

To understand the challenge of self-organization in relation to time let us have a look at a very important catalytic process: water splitting and oxygen evolution via the 4-centre manganese complex in photosynthesis. It is well known that it was photocatalytic water splitting, starting approximately $3.2-3.5$ billion years ago in blue-green cyanobacteria (Blankenship, 2010), which began generating our favourable climate and made higher life possible. If we could copy it technically with a cheap catalyst, solar generated fuel would become easily available 


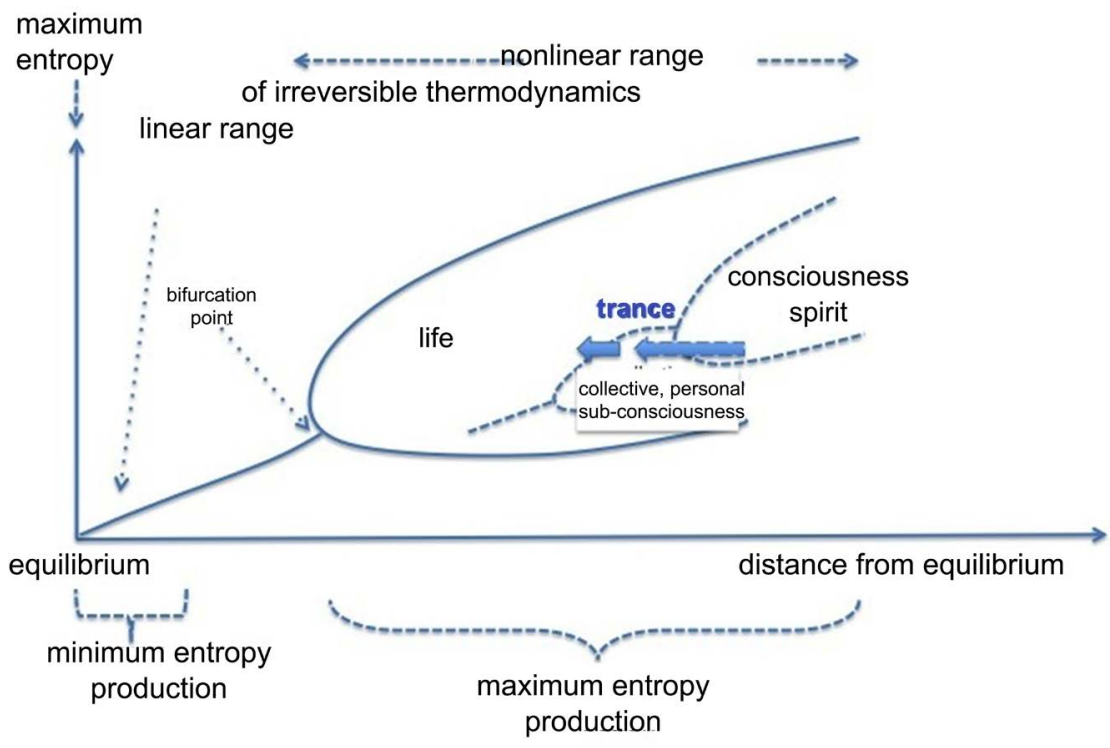

Figure 2. Scheme explaining life, consciousness and spirit as a function of distance from equilibrium. Also the presently accepted entropy laws, maximum entropy at equilibrium, minimum entropy production in the near equilibrium linear range are indicated, besides of the here proposed maximum entropy production in the nonlinear range of irreversible thermodynamics. Consciousness and spirit require high energy flow and distance from equilibrium. With decreasing energy supply consciousness is breaking down yielding trance-like states and rudimentary forms of consciousness (arrows).

and cheap, which explains many scientific efforts in this direction (e.g. Lewerenz \& Peter eds., 2013; Chen et al., Eds., 2013; Tributsch, 2008a).

What is the main problem with water splitting? In order to liberate one molecule of oxygen a catalyst has to extract 4 electrons from water. The minimum required energy would be 4 times $1.23 \mathrm{eV}$ plus some losses. However, when a first electron is extracted from water already $2.8 \mathrm{eV}$ are required and an $\mathrm{OH}$ radical is formed, which is not desired as an intermediate. An example of such a catalyst, working with UV-light, is titanium dioxide, which is now used to fabricate self-cleaning interfaces (Fujishima et al., 1999). But also when a platinum electrode is applied, a quite unfavourable mechanism of oxygen evolution proceeds. Nature, however, found a way to extract all four electrons from water with negligible losses of only $60 \mathrm{meV}$ per electron (Watanabe et al., 1990). In contrast, the best presently known artificial catalyst for oxygen evolution is the noble metal compound $\mathrm{RuO}_{2}$ with a loss of $300 \mathrm{meV}$ per extracted electron. But a remarkable fact is that in spite of many efforts no synthetic 4-centre manganese complex tailored to match the photosynthetic original, was found to show bio-analogue favourable catalytic properties (a review from 2004 already discusses 171 manganese complexes including $54 \mathrm{Mn}_{4}$ clusters, on the basis of 410 references (Mukhopadhyay et al., 2004). What is nature's secret? How can it make structurally equivalent complexes, which science finds essentially inactive, highly active? Already 25 years ago the problem was addressed (Pohlmann \& Tributsch, 1993) and it was suggested that in nature one is dealing with a 
self-organized molecular catalytic reaction. This is not a farfetched assumption because most structures in biological systems are self-organized. The $\mathrm{Mn}_{4}$-complex in photosynthesis accumulates stepwise four positive charges until the $\mathrm{S}_{4}$ state of the complex is reached (Kolling et al., 2012; Rhee et al., 1997). This state starts reacting with water and here feedback processes become crucial. When the first electron is extracted from water there will be, via some molecular reaction steps, a feedback to the next electron extraction step and from this to the following and so on. Mathematically it is then seen that under certain parameter constellation one equation only is sufficient to describe the transfer of all four electrons from water with low activation energy. This means that the first electron is "slaving" the others, increasing order and decreasing entropy (like what photons do in a laser pointer). As a consequence, the activation energy is seen to decrease dramatically and could even become negative (Pohlmann \& Tributsch, 1993). The unfavourable first electron extraction is avoided and the collective reaction becomes fast and highly directed. This is exactly what is seen to happen with the manganese complex in nature. But a key condition is that the protein environment of the manganese complex facilitates such feedback processes. This is an important detail, which is not yet adequately considered in present water splitting research (Tributsch, 2018a). And the feedback processes involved during self-organization definitely require a "before" and an "after" in time. What time? How can the $\mathrm{Mn}_{4}$-complex, on the basis of time-neutral particles, "know" how to react during feedback situations? Can we imagine that an efficient molecular catalyst, in which the overall reaction proceeds in the millisecond range or faster, relies on and monitors a probabilistic, statistical time arrow in the environment, a time arrow towards increasing entropy (disorder), to determine a "before" and an "after"? Such a statistical time arrow in direction of entropy increase would, on the basis of established knowledge, rely on a huge number of statistically reacting particles to function. What statistical disorder within and around the molecular complex, which itself is reducing its entropy and building up order during the self-organization process, would it be? How could a statistical time arrow be experienced by the manganese complex anyhow? How could a monitoring of the time flow for the fast proceeding self-organized molecular catalytic reaction proceed to allow distinction between "before" and "after"? On molecular level this is not imaginable to occur on the basis of a probabilistic time mechanism. But it is imaginable, when energy turnover itself, by generating action, is indicating the pulse of time. Even our clocks do not monitor an increase in statistical disorder. They rely on an energy input and special energy driven mechanisms to measure a flow of action, energy times time, from which our clock time is extracted via calibration.

During water splitting the process of time-finding must proceed within the catalyst molecule, which follows straightforward energy conversion steps. It must be related to the turnover of energy and the flow of action during the catalytic process. The flow and pulse of action will determine what was "before" and 
what will be "after". It is argued (Tributsch, 2016a) that time cannot be a statistical phenomenon, it must be fundamental, driven by energy turnover. Figure 1(b) explains how such an energy driven time arrow can be imagined. Energy is not any more time-neutral, but gets a fundamentally dynamic property and generates action. Many energy conversion steps, which are schematically shown in Figure 1(b), generate a flow of action (e.g. trickling sand in an hourglass), which can be calibrated to yield time. Here feedback processes during energy conversion processes can occur.

Such a fundamental time flow, an energy driven time arrow, will, of course, be equally needed for explaining the wealth of other biological mechanisms, of population dynamics, and of the process of biological evolution in general. The expectation is: only when accepting such a reality of an energy driven fundamental time arrow we will be able to learn how to construct working bio-mimetic models for photo-catalytic water splitting (which could be essential for human's energy future) and to understand relevant laws determining evolution of the universe and of life within. When trying to understand self-organized biological processes we have to think in terms of a time generating mechanism, which actually controls the biological, structure forming processes. We cannot use a time concept based on statistical probability towards disorder for understanding mechanisms in which energy flow and time control is required for building up order. This motivates and outlines the aim of this investigation: While historical and present understanding of evolution is based on a probabilistic time arrow, on energy which has no drive, and on ignorance of how self-organized systems far from equilibrium may behave in terms of entropy (energy) turnover, the present paper introduces an energy driven time arrow, which allows feedback processes. It explores the now deducible limiting entropy (energy) law for self-organized systems, and investigates consequences for understanding mechanisms of evolution. The principal interest is a re-evaluation of the situation of humans, subject to such a new proposed energy driven time arrow in a fundamentally irreversible universe.

\section{Deriving and Understanding the Fundamental Energy Driven Time Arrow}

\subsection{The Principle of Least Action Shows the Way}

When a stone, or a wheel spider, Carparachne aureoflava, are rolling down a hill or a sand dune respectively, selecting a variable speed and a special pathway depending on the geographical features encountered, they follow the principle of least action. Action, the time integral over energy turned over, is minimized. Most physical laws, even the general theory of relativity, have been linked to this principle, which, for three hundred years has been in the focus of physical, philosophical, mathematical and even esoteric considerations. Why did nature adopt this principle and why does the stone know where to go? The example of the stone seemed to suggest that physical systems are governed by final causes. It 
also became never clear why scalar, non oriented quantities, such as energy and time, as they are presently used to calculate least action, succeed in describing dynamic motion, which is a standard procedure in physics for calculations of ballistic trajectories or satellite orbits.

When action, the time integral over energy, is split up into infinitesimal small sections, then these still have to minimize in order to fulfil the principle's claim of minimization of action. This is, however, only possible, when the quantities involved, energy and time, are themselves dynamic quantities. Free, available energy, however, as it is understood now, is a scalar quantity, just a number without interest in changing and doing work. And time is just a parameter, monitoring changes. When these quantities do not have directed, vectorial properties, how can then the least action path of a stone be calculated? This is accomplished with a mathematical trick: the so called "variational calculus". Mathematics is achieving what we are not allowing energy and time to be: dynamic variables.

In contrast, as suggested in (Tributsch, 2016a), one should claim that energy and time are dynamic quantities, with the tendency of energy to decrease its presence per state, thus generating entropy while driving time. This also simply explains the final cause, which seemed to govern physical systems subject to the principle of least action. The principle of least action is just demonstrating a fundamental energy driven time arrow. It is a dynamic energy, which decreases its presence per state and thus generates entropy and drives time. This is, in principle, exactly what the empirical second law of thermodynamics is saying: that entropy is maximising in a closed space (which could not be derived from basic principles up to now) and what makes snowflakes grow (a minimum free energy is approached). Already these empirically observed important phenomena, which cannot be derived from time neutral basic principles, make the proposal of an energy driven fundamental time arrow credible and necessary.

Energy turnover drives time, but the real "essence" of time is not the parameter $\mathrm{t}$, clock time, which we use to monitor changes. It is rather the flow of action, energy times time, as it follows from the principle of least action. Even clocks are powered by a flow of action, from which a calibrated clock time $t$ is deduced. But clock time $t$ itself has no substance and there is no energy behind. Clock time cannot directly be measured like a stream of air or of water and it should not be able to manipulate objects and space (as assumed in Relativity Theory). It has always to be measured and handled via a flow of action (as described by the principle of least action, and implemented in a clock), which is the actual time flow in nature.

What does this mean in practice? Let us chose a relevant example. If we want to find out how life behaves on a planet in a fast moving galaxy far away we should not calculate (transform) time and energy separately for this planet, as done in the Theory of Relativity (yielding a time which is an illusion). We have to calculate the effect of relative movement on action, energy times time. And 
action remains the same. Life there may consequently proceed like it proceeds on our planet. Time travel, which follows from the Theory of Relativity, and would create so many incredible paradoxes, is simply not possible.

The claim here, that time flow is fundamental and energy driven, means a paradigm change. Our world is, on the level of atoms and elementary particles (which are equivalent to energy and mean self-organized energy (Tributsch, 2015; 2018a) not fundamentally time neutral, as it is claimed now, but fundamentally time-oriented and irreversible.

This conclusion has significant consequences for understanding physical mechanisms ranging from quantum physics to evolution of life and cosmology (Tributsch, 2015; 2016a, 2016b; 2018a). In quantum physics, for example, particle and wave concept are not equivalent (and time neutral) any more, but a wave, with its distributed energy, follows from a particle, with its concentrated energy. For a quantum state, with both particle and wave properties, information on matter is needed to recreate a particle from a wave. This would work like seen with a modern three-dimensional printer, which creates a structured object from information. The information on matter is part of a quantum state and turned out to be relevant for a "rational" understanding of quantum phenomena (Tributsch, 2016a), which itself should be a discussion point for science philosophy. Information on matter has an energy content, is localized around a particle and increases with particle numbers. It could be identified with the phenomenon of gravitation (Tributsch, 2016b). Since a particle is reconstructed via this information on matter (like an object can be reliably reconstructed via a 3D-printer from digital information transmitted to a moving plane), an always constant light velocity immediately follows. Such a simple and reasonable explanation of gravitation and of the always constant light velocity questions, of course, the generation of these phenomena by empty space (as postulated in the Theory of Relativity).

Due to the ongoing exchange between particle and wave, via information, in cosmology a spreading radiation of photons can lose energy for entropy formation, as required by the well known entropy law, which also Einstein used and compared with the entropy law for an expanding gas to derive the photon property of radiation (Einstein, 1905). For the reason of entropy generation alone stellar radiation should be experiencing a redshift, which is the stronger the longer the distance of the cosmic object. In quantum physics, as understood today, such entropy formation is not permitted. A photon, once released, can only change its energy when interacting with matter and gravitation. Therefore, an explosive spreading of empty space is presently assumed in physics to explain most of the observed stellar redshift (cosmological redshift). But how can empty space, defined to contain nothing, assume such a property of expanding and where is the missing energy going?

A first screening indicates that, accepting an energy driven fundamental time arrow, major paradoxes and implausible assumptions in quantum physics and 
cosmology can be avoided and replaced by rational explanations (Tributsch, 2016a, 2016b; Tributsch, 2015). It is claimed here that they arise in present physics because scientists look at an irreversible world applying laws deduced for a world with assumed time neutral fundamental properties (for philosophy a case of counter induction). And, as to be claimed and explained here, evolution does not fit into a time neutral world either. Our present concept of evolution has consequently to be re-evaluated.

\subsection{Statistical Time Arrow Is Only Specialized Manifestation of the Fundamental Energy Driven One}

Those phenomena attributed to the "statistical" time arrow actually exist. An "ordered" gas (compressed, assumed time-reversible) indeed becomes an expanded, statistically distributed, chaotic one (Figure 1(a)). The distribution of gas particles becomes a more probable one with less information content. The entropy $S$ of this gas, thereby, following the statistical time arrow towards increasing entropy, grows from $S_{1}$ to $S_{2}$ by $\Delta S$. When multiplying the entropy with the temperature $\mathrm{T}$ of the system, however, one realizes that this means, that the not anymore useful (entropic) energy of the system is simply increasing by $\mathrm{T} \Delta \mathrm{S}$. Since energy cannot be created from nothing and cannot be lost ( $1^{\text {st }}$ law of thermodynamics) this means that the statistical time arrow is simply explaining that original free (available) energy, contained in the more ordered system, is being converted into non available (entropic) energy of the more disordered system. Instead of saying that a system assumes a more probable state of disorder (compared to a time-neutral initial state) it is therefore entirely equivalent saying that (original) free energy has the tendency or drive to decrease its presence per state while generating entropic, not available energy $(\mathrm{T} \Delta \mathrm{S})$. Such a phenomenon is actually observed, for example as reason for the growth of ice crystals, and can accordingly be interpreted without claiming time-neutral gas particles (or time neutral energy).This however is exactly the proposed definition of the "fundamental" energy driven time arrow (Tributsch, 2016a). Energy and the resulting action (from which clock time can be derived) can therefore be understood as dynamic variables. These simple considerations are, besides of the conclusions derived from the principle of least action, another support for an energy concept, in which energy is not dormant, but dynamic. It can also generate statistical disorder (simulate a statistical time arrow) while converting energy and producing action and entropy.

This finding, that established facts in relation to the statistical time arrow are not in contradiction with the proposed concept of a fundamental time arrow, but easily incorporable into it, is important. Present concepts and knowledge can be considered to describe special, limiting cases. They can thus be incorporated into the concept of an energy driven fundamental time arrow. One can go on using many tried and tested existing theories from physics and physical chemistry, but with the restriction that they must not be fundamentally coined by irre- 
versible mechanisms.

There is, namely, a big difference between the two time concepts: While the statistical, probabilistic time arrow does not allow to understand self-organization (via feedback processes) in highly organised fast reacting molecular structures and in elementary particles, the fundamental time arrow does. It is the flow of action as a result of energy conversion, which is determining the progress of time. The fundamental time arrow, related to a dynamic energy, is much deeper rooted and more general than a probabilistic, statistical time arrow. And before all: it is the time, which is actually relevant in natural processes and determining molecular self-organization, evolution, population dynamics and cosmological phenomena. Considering it is crucial. It will allow understanding nature, and especially mechanisms which depend on time, feed-back and self-organization, in a more rational, simpler and deeper way.

Energy turnover, the flow of action and the build-up of order are fundamental characteristics of biological and human evolution. In the following some important consequences of a fundamental time arrow for biology, evolution and population dynamics should be investigated.

\section{What Entropy Law Is Determining Self-Organized Systems Such as Life?}

Our traditional science cannot convincingly derive the empirical second law of thermodynamics from fundamental principles, the well-established law, which states that in a closed space entropy, disorder, increases and maximises. Additional ad hoc assumptions would be necessary. For physics, which claims that elementary particles and processes are time neutral, this is a humiliating fact. But when the discussed fundamental time arrow is accepted, with an energy, which aims at decreasing its presence per state and drives time (like during the growth of snow crystals), the second law of thermodynamics follows immediately. It allows understanding and deriving the entropy law for equilibrium thermodynamics and is an important result which alone justifies taking the fundamental energy driven time arrow seriously.

Life is a phenomenon of self-organization, which is proceeding far from equilibrium in the range of nonlinear irreversible thermodynamics. Its position in a diagram explaining systems exposed to different distances from thermodynamic equilibrium is shown in Figure 2. Also the known entropy laws (maximum entropy, minimum entropy production) for equilibrium and near equilibrium, and the new proposed entropy law for self-organized systems (maximum entropy production, see later) are indicated.

Feedback processes are fundamentally involved in building up order. This order is generated at the expense of overall entropy production. What entropy law applies for life far from equilibrium? Present physics and thermodynamics has no answer. Such a law cannot be derived. But with a dynamic energy that decreases its presence per state and drives a fundamental time arrow the situation 
is different and quite clear: such self-organized energy systems, which are powered by the through-flux of energy, will finally transform all available energy by decreasing its presence per (energy) state thereby producing entropy. Feedback processes, which cycle products again into initial reactions and amplify them, will fuel the self-organization phenomenon, increase the energy turnover and consume the available energy influx, intensifying the process, when more energy becomes available."Maximum entropy production" is the determining mechanism of feedback driven processes and only limited by the constraints of the system, by the limitations, which the system itself or the environment are imposing. It is interesting to note now that maximum entropy production within the constraints of the system has already been empirically proposed by other scientists for self-organized systems including human ecology (Paltridge, 1979; Swenson, 1997; Liu et al., 2011).

Empirical evidence has been given by these authors, but they could not justify such an entropy law and derive it from more fundamental principles. In addition, they met strong objection from experienced physical chemists. John Ross wrote a paper on the "invalidity of the principle of maximum entropy production" and argues that the rates of chemical reactions are controlled by (non-vectorial, non-oriented) thermodynamic quantities such as Gibbs free energy and activation energy and not by the rate of entropy production (Ross, 2008). His arguments are certainly valid, however for the traditional handling of energy as quantity of state without a time orientation. If energy is however understood to be a time oriented, dynamic variable aiming at decreasing its presence per state, and is thereby indeed generating entropy via feedback controlled mechanisms in a rate determining process, the situation changes. Self-organized systems do therefore aim at maximum entropy production, of course within the constraints of the system concerned. Then, published empirical observations in support of maximum entropy generation make sense (Paltridge, 1979; Swenson, 1997; Liu et al., 2011).

There are practically interesting examples of self-organized system, which will allow us to imagine how life may behave, how population dynamics may work, when such a law of maximum entropy production applies and constraints are changed, for example reduced:

A well known inorganic phenomenon of a self-organized system, which is causing many problems to humans, are hurricanes. Many of these quite frequently arising storms remain relatively small and modest. When they hit land they also typically lose power and disappear.

Sometimes, however, a hurricane (cyclone in the Pacific) grows within a few days to larger and larger proportions and becomes a super-hurricane of category 5 (e.g. Patricia (Pacific, 2015) with $345 \mathrm{~km} / \mathrm{h}$, Katrina (Atlantic, 2005) with 280 $\mathrm{km} / \mathrm{h}$, Maria (Atlantic, 2017) with $280 \mathrm{~km} / \mathrm{h}$ ). Science does presently not yet understand exactly why some hurricanes become big and others stay small. How big can such storms become? A much bigger storm than ever seen in the atmos- 
phere of the earth is occurring in the atmosphere of Jupiter (where no continents can be identified) and seen as red spot on its surface. Its dimension extends over 22,000 times $12,000 \mathrm{~km}$ and it already has been raging for centuries. It is so violent that the temperature above the spot on this cold planet reaches $1300^{\circ} \mathrm{C}$. Maximum entropy production within the constraints of the system would perfectly explain the self-organized behaviour of such atmospheric storms. They tend to become as big and as violent as the constraints of the system allow it. Within their constraints they generate maximum entropy turnover.

Let us now look at a laboratory example of a self-organized system and focus on its ability to adapt and evolve towards additional energy sources. It is an oscillating chemical reaction, the Belousov-Zhabotinsky reaction, which, with its beautiful propagating rings and waves, is often used as a relatively simple laboratory demonstration for the function of (much more complex) biological self-organized systems (compare Wikipedia). If the system would be scaled up, while maintaining the parameters needed for sustaining oscillations, oscillating energy conversion would expand and proceed, adjusting itself to higher energy turnover, until all the additional chemical energy (malonic acid) is consumed. How does such a system respond, when additional energy, for example in form of light, is made available? As a consequence of addition of the light absorbing dye tris (bipyridine)ruthenium(II)chloride, which can supply photo-excited electrons to the system, the self-organized reaction adjusts and oscillation patterns can now be controlled by light. The self-organized system adopts a photoreaction and readily learns to utilize light, solar energy (!). If a light pattern is now projected onto the oscillating solution, it is clearly reflected and recognized in the correspondingly adjusted oscillation patterns. Chemically, energy conversion via an electron transfer process, occurring in the dark, was completed and replaced by a light-induced one, providing more and a different form of energy to the system. When looking at the introduction of the photochemical process (via addition of a dye molecule) as a "macro-mutation", then the system simply adjusts to higher energy turnover and entropy production (maximum entropy production within the new restraints of the system) by using light energy. It simply allows additional energy into the system as long as existing restraints allow it. That way it becomes, as a photo-chemical reaction, more complex and differently self-organized. This shows that self-organized systems can, when additional energy is made available, evolve towards higher complexity and higher energy consumption. The complicated feedback processes involved in this case (at least 12 kinetic differential equations needed for a mathematical description) presuppose a time flow, a distinction between "before" and "after". As explained earlier, it cannot be a probabilistic time, but must be a time dictated by energy conversion steps involved in this complex reaction, which generates order within this oscillating chemical system.

As a third example let us look at biology and select trees. They can be dwarfs or very big such as Eucalyptus or Sequoia, which can become more than 100 
metres high. Trees use solar energy via quantum processes and solar evaporation for photosynthesis and tensile water transport. They generate useful work via massive entropy production in form of environmental heat and water vapour. Trees are definitively systems which function via self-organized processes subject to maximum entropy production (energy turnover), within their restraints, which largely vary. They are, of course, genetically controlled, which shows that genetic mechanisms had to deal, and successfully dealt with and had to "respect" the fundamental properties of self-organized systems (maximum entropy production within existing restraints). Living systems and genetic control evolved within the basic conditions imposed by self-organized systems. Genetic mechanisms developed and acted towards the evolution and diversification of self-organized systems, which, however, remained subject to the fundamental entropy law, governing them. Only within its characteristic entropy law life as a self-organized system remained operable.

\section{What Consequences for Understanding Evolution?}

\subsection{Early Evolution towards Light Harvesting}

Today there is agreement that evolution follows no purpose, is blind and indifferent, just exposed to statistical mutations, which epigenetic mechanisms can to some degree influence. But this established concept ignores, how self-organized systems themselves deal with time in feedback reactions and with energy. Traditional theory of biological evolution emphasizes genetic mechanisms, but essentially excludes the influence of fundamental laws which control self-organization (which physics and thermodynamics could not identify on the basis of time-neutral laws and a probabilistic time arrow).The before mentioned example of the Belousov-Zhabotinsky reaction shows, how easily a self-organized mechanism integrates light absorbing molecules and a photoreaction towards solar energy utilization and a maximization of entropy (energy) turnover (within the new restraints of the system). All available energy is finally converted into entropic, not any more available energy $(\mathrm{T} \Delta \mathrm{S}$, with $\mathrm{T}=$ absolute temperature, $\Delta \mathrm{S}=$ entropy change). Since a similar step must have happened during early evolution of photosynthesis, which occurred between 3.2 and 3.5 billion years ago, when life started using solar energy, it is reasonable to have a closer look at it. Life itself, of course, started earlier and a credible hypothesis is, that this happened around black smokers in the deep sea on the surface of pyrite mineral (Wächtershäuser, 2000). In favour of this model is not only that life (bacteria) can draw chemical energy from pyrite, iron disulphide, but also that iron-sulphur complexes (components of pyrite) have survived in all biological systems as ferredoxins, and act as relevant catalytic and electron transfer centres. In addition, in presence of the amino acid cysteine, pyrite can be dissolved in form of small cysteine-stabilized iron-sulphur clusters, which can serve for energy harvesting. But since photosynthesis required visible solar light, life must have continued evolving also close to the ocean surface in tidal regions. A problem, how- 
ever, was that due to the then missing ozone layer the UV irradiation there must have been 100 times stronger than today. How could early life have nevertheless survived there? The explanation, proposed by the author, is that pyrite can also serve as an excellent energy acceptor and quenching agent, via non radiating energy transfer, of excitation energy of molecules at a distance within approximately $10 \mathrm{~nm}$ (Tributsch et al., 2003; see also Mullen, 2004). In pyrite, as the final acceptor of this UV excitation energy, the excitation state is quenched within $10^{-14} \mathrm{~s}$, converted into heat. As a consequence, evolving life in a thin layer on pyrite was reasonably protected against radiation damage. Here the "living" organic layer could have started first taking advantage from a photovoltaic effect, which has been demonstrated for natural pyrite. It would have been like replacing dark electron transfer by photo-induced electron transfer in the Belousov-Zhabotinsky reaction when introducing a dye. In such self-organized thin organic layers on pyritemineral surfaces in tidal areas of the ocean Chlorophyll-like dye molecules may have appeared. They started interacting photo-chemically, contributing radiation energy into the "energy-hungry" self-organized early living system, which then gradually separated from pyrite and continued increasing local order at the expense of solar energy. The entropy law identified for self-organized systems explains the feedback-driven "pressure" towards maximum energy turnover and towards mechanisms facilitating it. All available energy for the self-organized system is turned over in direction of dissipation and entropy formation (of course within the restraints of the system). A higher energy through-flux will shift the system further away from equilibrium. This can facilitate additional build-up of order. Early evolution of photosynthesis, as analysed in detail in the literature (Blankenship, 2010), could proceed its way. But somewhere during the early stage of life genetic mechanisms must have been integrated to take control over reproduction and evolution including life's sometimes critical and destructive exposure to maximum entropy (energy) turnover (see later). Formation of self-similar copies is a characteristic property of self-organization and self-organization of chemical DNA information (as to be explained below) will provide the necessary mechanism.

\subsection{Opportunities for Endosymbiosis and Macro-Mutations}

Evolution of early light harvesting mechanism via the described property of self-organized systems of maximising energy (entropy) turnover in combination with genetic mechanisms is easier understandable than an evolution entirely by chance and via statistical micro-mutations, random copying errors, without aim (just imagine the complex photosynthetic mechanism developed via statistical mutations only).The trend towards maximum entropy (energy) turnover gives access to relevant energy related options. Such boundary conditions clearly favoured endosymbiotic mechanism, such as the symbiosis of prokaryotes, of bacteria and archaea, as well as the incorporation of energy converting mitochondria and chloroplasts into eukaryotic cells, which happened 1.5 billion years ago. 
The processes just followed the here discussed entropy (energy) law of self-organized systems. The evolving systems simply acquired new energy sources (as explained for the Belousov-Zhabotinsky reaction with the incorporation of a light harvesting mechanism). This also opens the consequence that mutations now have a bigger chance to succeed. The reason is that more energy is available, which helps them to be more successful (like ideas in industry are more successful when sufficient money (energy) is available). In addition, the increasing energy turnover can give access to new self-organization structures, because the system can move further away from equilibrium (compare Figure 2) towards new bifurcation opportunities.

Such fundamentally tolerated and imposed mechanisms could play a role in explaining significant shifts and reorientations within evolution (endosymbiosis and macro-mutations via adaptive radiation), which started new species. But they, of course, required genetic mechanisms to participate, in order to perpetuate information and to guarantee that the complex parameter space, which sustains self-organization, is not left.

Self-organized systems, subject to maximum entropy (energy) turnover, have an inherent drive to facilitate evolution towards higher complexity and higher energy turnover. As long as the system can survive and control order within its special parameter constellation, it will allow entropy turnover to increase. The energy hunger is inherent. But the adjustment to the right parameter constellation, which facilitates and propagates self-organization, can be expected to be a great challenge. The essential point is: maximising energy (entropy) turnover shows a direction of evolution, explains the energy hunger of living organisms, but it does not yet shape a living and multiplying organism. Here, of course, the complex mechanisms of selection and evolution enter, as science has learned to understand them from sometimes somewhat different points of view (e.g. Novak, 2006; Okasha, 2006; Lynch, 2007; Futuyma \& Kirkpatrick 2017). For real life to develop, the drive towards maximum entropy production, as applicable to self-organized mechanism, had to be superimposed by the evolving information carrying and selecting genetic mechanisms (which is also subject to self-organization, as explained further below).

\subsection{Maximum Entropy Production of Self-Organized Systems and Expected Overall Trend of Evolution}

Living systems are not only self-organized but are simultaneously open systems. Energy is flowing through them and they are competing among each other and interacting with other self-organized systems (other living systems, climate, ecosystems). Uncontrolled, they will essentially behave like atmospheric storms or the Belousov-Zhabotinsky reaction. They may suddenly die down or they may tend to maximise their energy turnover, whenever possible and within their restraints. A problem for life was to maintain the system within the parameter space, which sustains order. What became the most important guiding restraints for evolution of species, the restraints, that helped selecting the appropriate ad- 
aptations on the way towards increasing energy turnover? Life, as we know it, would not have survived, if increasing energy (entropy) turnover would have been the only determining factor. Not considering reproduction now, earlier or later self-organization and build-up of order would have broken down, because the systems would have drifted out of the narrow parameter space, which sustains self-organization. The evolutionary process of statistical mutations and adaptations towards fitter living species (e.g. Ruse, 1996; Futuyma \& Kirkpatrick 2017) must have taken over to safeguard and shape the survival ability. It is, however, to be expected, that the trend towards increasing energy (entropy) turnover of self-organized systems essentially shaped evolution because living systems with their genetic mechanism do not stop being self-organized. Evolution would be like introducing small and arbitrary changes into the self-organization patterns of a hurricane or the Belousov-Zhabotinsky reaction. The fundamental drive towards increasing energy turnover is expected to be maintained, to favour macro-mutations and to explore new self-organization patterns (which could improve the survival ability of species).But this is expected to have been a delicate process. As a consequence, it has to be assumed that DNA and the genetic code may not only have evolved for handling, transfer and multiplication of information between subsequent generations. The genetic tools may have also evolved to safeguard life against an uncontrolled maximization of energy turnover imposed by the very nature of self-organised living systems. The danger to drop out from the parameter space, which can sustain self-organization, was quite big. This situation may be characterized as follows: evolution, since it deals with self-organized systems, is subject to a natural law that drives them towards maximum entropy (energy) turnover (within the restraints of the system). But genetic control (self-organized, see later) and statistically occurring mutations are superposed to such a drive, handle reproduction, avoid destructive maximisation of energy turnover, and find the way to keep life in the appropriate parameter spaces for survival. But a mutation is not only an arbitrary statistical change during reproduction. It becomes also a modification within the self-organized system itself. And such a modification can trigger changes in self-organization and in the restraints of the system. This would, of course, also contribute to evolution. The result is what was above mentioned as an example: trees. They are genetically controlled but show the property of maximum entropy production (energy turnover) within their restraints (different sizes, different climatic adaptations, different water needs).

That genetic mechanisms can also act towards a control of excessive energy turnover is obvious. Many living species are experiencing this during periods of hibernation and sleeping. Here, genetic mechanisms care for a reduction of energy consumption of living species as an adaptation to reduced energy supply. That way the parameter space for self-organization (life) could still be sustained.

Self-organized irreversible processes and genetic processes (also self-organized, see later) can, in addition, interact and synchronize. This is generally known for self-organized systems, but how this could work in detail has still to be found out 
for evolution. It is to be expected that different restraints apply for the long-term, genetically controlled evolution of individual species and for much shorter term population dynamics.

It is interesting to look at simpler examples of self-organized systems to understand more about their inherent properties. How they behave when they are not subject to a longer term genetic or external control, can best be seen from examples of population dynamics. Self-organized systems, succeeding in a special environment, will increase their energy turnover and adjust it to the new constraints. The outcome can be temporary but is still impressive. An example is the population dynamics of imported rabbits in Australia. Their energy consumption devastated large landscapes until they were more or less controlled by external restraints. Another example is the Gipsy moth, Lymantria dispar dispar. It appears in a chaotic rhythm, many years inconspicuously, but then in an explosive manner, generating maximum entropy turnover. This chaotic behaviour is a possible, typical reaction pattern for a feed-back controlled self-organized mechanism. A further example is sheep, when developing uncontrolled on islands. The ecosystem of Santa Cruz Island, one of the Channel Islands off the Californian coast, was practically destroyed, with a peak of 130,000 sheep and 5000 pigs living wild, before radical conservation measures were initiated towards creation of a National Park. These examples show how uncontrolled self-organizing systems behave on their way towards maximum entropy production.

When an adequate restraint (e.g. genetic control) is superposed, there will still be a pressure and trend in evolution towards increasing energy turnover, because this property is fundamental, explains the energy hunger of living systems and may pioneer relevant adaptations. But evolution is expected to be much more focussed and will be more safely guided through varying environmental situations, because unfavourable mutations will simply be eliminated. For individual species there is consequently still a long-term trend in evolution of life towards higher energy turnover to be expected. Can this be recognized in nature? There exists, for example, an evolutionary trend towards multi-cellular organisms and higher energy consumption via a larger size. The biggest living creatures did not live during the time of dinosaurs but they are living now, not accounting for the prehistoric extinction of many other large animals, by humans (e.g. Camelops spp., Mammut americanum, N-America; Mammuthus primigenius, Coelodonta antiquitatis, Bos primigenius, Eurasia; Diprotodon optatum in Australia, Megalapterix didinus in New Zealand; Aepyornis maximus in Madagascar). There is the blue whale, (Balaenoptera musculus), which reaches a length of $33 \mathrm{~m}$ and a weight of 200 tons. The biggest tree, Sequoiadendron giganteum (General Sherman tree in Sequoia National Park, USA) is 83.8 $\mathrm{m}$ high, has a circumference of $31 \mathrm{~m}$ at its base and a volume of approximately 1500 cubic metres. A plant fungus of the Physalacriaceae family, a specimen of Armillaria ostoyae, which was found in the Malheur National forest in Oregon 
covers an area of 9 square kilometres and is estimated to weight approximately 600 tons. The bigger a creature, a self-organized system, the more energy is turned over and consequently the more entropy is generated. This is also true for the growing human world population.

When more energy is turned over, systems are pushed further away from equilibrium. This favours, in self-organized systems, a change and increase of order via new self-organization patterns. Evolution of species should consequently also show a clear trend towards higher order and perfection, from simple unicellular to more and more sophisticated multicellular living beings and symbiotic associations. The latter will simply be experienced as a source for additional energy, supporting the trend towards maximum entropy (energy) turnover, within the (new) restraints. Also, significant organelles and organs in living species may have been incorporated through endosymbiosis or developed because of their role in energy turnover towards maximum entropy production.

Paleontological studies show that there is such a trend towards higher perfection. Living systems of today are technically more advanced than those that existed a few hundred million years ago. They also consume more energy. A human from a primitive hunter-gatherer tribe has a power consumption of approximately 100 Watt, 10 times more than the power consumption of a coldblooded crocodile of similar size. The power consumption of a modern human is 60 to 120 times higher than that of a primitive human. The use of fire, coal, oil and gas has helped him to follow the trend of self-organized systems and people also tend to increasingly crowd in cities, because more opportunities (energy) are available there.

With more energy available there should also be more implemented mutations. The rate of mutations should therefore have increased towards present time. They act like innovations in a technical society, which can be more successfully implemented when abundant money (energy) is available. An increasing rate of mutations towards present time seems actually to have been confirmed for evolution (Hawks et al., 2007). But, as the introduction of a light-inducted reaction into the Belousov-Zhabotinsky mechanism has shown, changes towards an increased energy through-flow via a new technology will simultaneously be favoured. Industrial inventions, "mutations", cars, airplanes, computers, cellphones, digitalized and automatic factories, all add to energy (entropy) turnover.

Examples of species, which evolved back during adaptive radiation to less energy consumption and smaller size, for example when isolated on an island, are of course also known (e.g. homo floresiensis). Their existence is not in contradiction with the proposed biological trend towards maximum entropy production within the restraints of the systems. The external restraints for such systems simply changed and reduced energy availability. They had to adjust to the deteriorating conditions. For other systems the constraints did not change during the last 200 million years. The solar light harvesting leaves of Gingko biloba, for 
example, remained essentially the same during this long time period. It made no energetic sense to increase the size of the Gingko leaf. On the basis of the existing tree architecture, the large number of leaves and their attachment there would not have been an advantage. In contrast, the leaves of the water lily Victoria amazonica could become bigger and bigger, because their constraints allowed them to harvest more and more energy. They could float on water, where they now reach a diameter of up to $3 \mathrm{~m}$ and a weight of $6 \mathrm{~kg}$. Also the leaves of the palm Raphia regalis could become large (up to $25 \mathrm{~m}$ ), because their anchoring allowed it.

What about humans, who, during the last centuries have been exploiting and "slaving" more and more plants and animals for energy consumption? They win competition with other self-organized systems and continuously reduce the constraints applied to their own self-organized system. The step towards globalization with the steadily increasing rate of global exchange of materials and energy is an example of developments in that direction. The fate of human civilisation, according to the deduced dynamic law governing self-organized systems, will be that more and more entropy will be produced. This means more and more energy turnover. More recent contributions to energy consumption arose from cell phones, computers, the Internet and the worldwide exchange of industrial products. Science fiction movies suggest how humans imagine the future: massive energy is turned over to sustain their living standard. Based on this materialistic interpretation human society will basically behave and go on like an atmospheric storm. It will increase energy turnover as long as the external restraints (and genetic control) permit it. Energy turnover will become bigger and bigger, because natural restraints for modern humans are (temporarily?) decreasing and humans learn to harvest more and more energy.

\section{Consciousness and Spirit}

\subsection{Subject to a Fundamental Energy Driven Time Information Can Self-Organize}

Generation and turnover of information involves energy (one bit is equivalent to $\mathrm{kT} \ln 2, \mathrm{k}=$ Boltzmann constant, $\mathrm{T}=$ absolute temperature). We know that matter can self-organize. We see this in living nature. Matter is related to mass, mass to energy. When (dynamic) energy flow is linked to time flow then information is consequently also able to self-organize. This can mathematically be demonstrated (Tributsch, 2016b). When a reasonable degree of complexity is given, so that feedback processes can be activated at a high rate, information modes within a nervous structure should be able to develop self-organization. However, the information processing system has to be pushed far from equilibrium, which requires that a high through-flux of energy through the brain is warranted (Figure 2). Another precondition would, of course, be that information on time, on "before" and "after" should be available on a very fast time scale to facilitate the crucial high rate of feedback processes. This requires a rapidly responding 
fundamental time arrow. As already emphasized, it is not imaginable that a statistical time arrow, relying on probabilistic events of many particles, as favoured today in physics, could do the job in a brain system. How could anyhow an increase of entropy, of disorder (in a system relying on building up order, thus reducing entropy) be monitored on a fast time scale? It must be, as here suggested, the flow of action as a consequence of energy conversion within the neural network of the brain, which controls and monitors the flow of time and which allows cascades of feed-back reactions to facilitate self-organization of information.

What does, in fact, self-organization of information mean? The consequence should be that a higher hierarchy of information processing is enabled. The difference in quality between pure information processing (like in our computers) and self-organized information processing (like in our brain) should be approximately the same as between water with the dissolved chemical components of a bacterial cell and a living bacterium itself. In the bacterial cell chemicals are handled in an "intelligent" way. The same can be assumed for self-organized processing of information. It may handle more basic information in an intelligent way and behave like a living system. Such a phenomenon can only be consciousness and human spirit. It may be considered as something, which is processing available information from memory, the body and the environment while creating a functioning model of oneself. Can animals do that? The question of consciousness in biological organisms is very complex. Intelligent questions have been raised in that context, last not least by the philosopher Nagel (1974), who argued, that then there must be something that is "to be like that organism". Nagel imagined a bat. Obviously, with such a definition, the information available on the particular living being should be provided and be available in a higher hierarchy of computation. This is exactly what should be expected from self-organized information. But there are additional conditions: The brain system must be driven for feedback processes far from equilibrium, which requires high energy turnover, and the species should show clear behavioural signs of thinking and acting in a higher hierarchy of information handling. Humans evolved a brain which is 3 times larger than those of the closest relatives among primates and show this consciousness.

How humans adapted to higher energy turnover in the brain which consumes up to $25 \%$ of the body's energy turnover while only accounting for $2 \%$ of the weight, is well known. Humans adopted an erect posture, lost body hair to better chase animal prey during hot hours of the day by releasing heat via sweating. More meat was consumed and cooking with fire allowed to better digest the food. A greater fraction of brain development was also postponed to a longer period after birth at the expense of longer child care and of social involvement of the human community. Human evolution indeed followed the way of self-organized systems towards maximum entropy production (within given restraints). 
Like with self-organization of matter in biological systems consciousness should have evolved gradually, forming earlier, more rudimentary and later more elaborated states of consciousness (compare Figure 2). Psychological science actually distinguishes collective and personal sub-consciousness (Jung, 1958-1981). They may function closer to equilibrium. Some primates as well may have already developed a primitive form of consciousness, but a clear evaluation seems not yet possible, especially since the phenomenon of consciousness itself is not sufficiently well understood.

Figure 2 shows, how life and consciousness developed far from equilibrium, the first via self-organization of matter, the second via self-organization of information (which requires and presupposes a certain degree of organization of matter). Higher energy turnover in the brain increases the distance from equilibrium and generates increasingly evolved self-organized states of consciousness.

The identified drive of evolution towards maximum entropy (energy) turnover within the restraints of the system has facilitated a significantly increased energy flow through the brain, which finally allowed self-organization of information leading to evolution of spirit and intelligence. It is important to emphasize here that the inability of traditional science to explain consciousness and spirit on the basis of established time-neutral natural laws has been seriously criticized. Thomas Nagel argued that the necessary preconditions for spirit must have been present in nature before human evolution (Nagel, 2012). The title of his book: "Mind and Cosmos. Why the Materialist Neo-Darwinian Conception of Nature Is Almost Certainly False" is quite provocative.In the present paper the explanation is easily given and it is entirely materialistic. It requires the here discussed paradigm change towards a fundamental energy driven time arrow and thus to a fundamentally irreversible world: "The preconditions for a development and for a materialistic understanding of spirit were present in nature in form of a drive towards maximum entropy production present in self-organized (living) systems, which are exposed to an energy driven time arrow. Genetic mechanisms sustained and developed spirit via natural selection". According to what we know about their brain and behaviour, largely cold blooded dinosaurs did not yet make it to develop spirit, even though nature experimented with very large body sizes to increase entropy production. But mammals much later succeeded in developing a brain with high energy throughput via the human species. Nagel (2012) argues that evolution itself cannot be justified via evolutionary arguments. But this ignores the here emphasized drive of self-organized systems towards maximum entropy production, subject to superposed genetic mechanisms (which, when self-organized, themselves turn over, multiply and maximize information, see below).This provides the drive towards survival and reproduction seen in evolution.

It is interesting to note that not biology generated the problem, which obscured understanding evolution of spirit in the past, but physics, claiming time neutral elemental particles and time neutral fundamental laws. They did not al- 
low understanding the entropy law, which is dominating self-organized systems and facilitating high energy turnover as a precondition for self-organization of information.

\subsection{Spirit as a Materialistic Phenomenon}

Subject to a fundamental time arrow, which is directly related to the turnover of energy and the flow of action within biological structures, consciousness, intelligence and spirit become natural materialistic phenomena. They are consequences of self-organization of information, triggered via a sufficiently strong through-flux of energy, which displaces the information system far enough from equilibrium (Figure 2). On the basis of known behaviour of self-organized systems several properties of consciousness and spirit may be immediately recognized. One is that consciousness will function only within well defined parameter constellations. If it leaves them, chaotic (break down), oscillating (manic depression?) or bi-stable (schizophrenic?) conditions may occur. Another factor will certainly be the supply of oxygen to the brain, which promotes energy conversion by serving as final acceptor for electrons. If oxygen supply is too low or when the energy flux through the brain decreases for other reasons, consciousness may be significantly reduced or lost, situations like trance may temporarily appear. This may be experienced in the altitude sickness, in ecstatic situations or in spiritual dementia. The operation conditions for consciousness would simply shift too much towards equilibrium and self-organization of information would drift into more primitive forms or entirely cease (arrows shown in Figure 2).

Since the self-organized information system, spirit, will involve many information sources, including those from memory, from sub-consciousness, from the living body, and the environment, it's very complex structure and dynamics will be significantly different from person to person. The created image of oneself, within "consciousness", and the resulting formation of intentions should therefore be very individual. To a large extent there will be a "free will". Due to the complexity involved it may not easily be possible to trace decisions back to trivial causes. But, on the other hand, self-organized systems can, under certain conditions, also become synchronized, maybe explaining mass psychogenic phenomena.

\subsection{Self-Organization of Chemical (DNA) Information}

Not only self-organized information in the brain, based on electrochemical processes, should work, but also self-organization of information, based on chemical information, the information coded in the DNA and the genome. Precondition, however, should be that also this information system is sufficiently pushed away from equilibrium via adequate through-flow of energy for self-organization. What speaks for such an idea? When the Human Genome Project was started with the intention to decipher the human genetic code the assumption was that the 2.9 billion base pairs in the genome will function as a 
blueprint for the body's structure and function and that it can properly handle and arrange the approximately 30 trillion human cells in the living body. However, a puzzling situation arose when the information storable in the human genome was estimated to be only of the order of 750 Megabyte, the storage capacity needed to operate a typical videogame (e.g. McElheny, 2012; Dharma-Wardana, 2013). Life is, of course, much more complex than a videogame.

What if the genetic code would only be a reference facilitating and shaping self-organization of its contained chemical information, while interacting with the living organism? Complex feedback processes would, of course, be needed for such a function. The self-organized living organism could provide that on the basis of a fundamental time arrow. A much more complex self-organized (chemically intelligent) information structure would result. A living body, into which modified genetic information is introduced, could facilitate the dynamics of self-organization using the genetic information as a reference system. A similar need for interaction has already been recognized between the DNA and the living organism within epigenetic concepts. Similar as the information contained in the structure and function of a living bacterial cell is much more extensive than that contained in its dissolved (not self-organized) chemicals, self-organized chemical information will be much enhanced but still largely determined by the DNA blueprint which defines the parameters involved. The system would not only be able to synthesize the necessary molecular biological building stones. It would also know how to arrange them and how to get them communicating with each other. It would be, superficially seen, like entering the (self-organized) Internet with keywords to retrieve and activate relevant and abundant information. The key-words would come from the DNA, the self-organized structure from a living, self-organized body.

Since information has an energy content, maximum entropy turnover, within the restraints of the system, would also apply for self-organized information handling. This means that maximum information turnover, within the restraints of the system, would be approached. The system would try to enhance the information content. It would multiply its information content and turnover by creating additional self-similar information. Fractal, self-similar structures are characteristic properties of self-organized systems. They are found in many biological structures (e.g. in the structure of cabbage). Such self-similar structures have consequently also to be expected, when information is self-organized. Information will consequently create self-similar information. This is, indeed, a characteristic property of life! The available genetic information is doubled and multiplied. Every new cell gets genetic information incorporated. The genetic code itself cannot easily explain that drive towards multiplication of information and why it was present at the beginning of evolution. Here it can simply be assumed that self-organization during early life included self-organization of DNA information. The result was self-similar additional information. It may have been the first step of a self-organized system towards reproduction. Self-organized 
chemical information handling thus helps to better understand living processes and may also shed new light on evolution of life on earth and in the universe. Evolution of "spirit and intelligence", as self-organization processes, both in neuronal and DNA systems, may become interesting new long-term research subjects.

In Section 4.1, it was shown how easily a new energy source (such as solar energy) could have been integrated into self-organized evolving life via incorporation of light absorbing molecules. Now, photosynthetic activities are all rooted in the controlling genetic code, which here is suggested to function in a self-organized way. What is the interrelation between the tendency of a self-organized system to maximize energy (entropy) turnover for locally increasing order, and the genetic code, which seems to be a quite fixed blueprint of life? An interaction may be less rigid, when both processes are considered self-organized. Self-organized systems can, as already well known from neuroscience (e.g. Izhikevich, 2007; Hesse \& Gross, 2014), get synchronized. This synchronization is also self-organized. Many examples from biology show that. Could this possibly indicate that increasing complexity due to increasing energy turnover eventually finds its way somehow into the (self-organizing) genetic code? Is this ability to synchronize another unexplored way to shape evolutionary pathways and maybe an additional opportunity to learn more about early evolution and the function of the genetic code when new species evolved?

In the here sketched situation the genetic code not only transmits information for reproduction, it may also safeguard life against uncontrolled maximisation of energy (entropy) turnover. An important task could be to respect and explore the restraints, which enable the survival of living organism, in relation to the trend towards maximisation of energy turnover. In the same way as the genetic system activates and controls energy limitations during hibernation and sleep in many species it could also control them during regular living activities. If environmental factors or a genetic defect relax such restraints in an unfavourable way, uncontrolled function or growth (as imposed by self-organized systems themselves) is then expected to occur in living organisms. One could speculate that this could be the phenomenon of cancer or of other severe diseases, which destroy a living organism. A simple question could now help to see whether such considerations are reasonable: what would be a straightforward way, within the presented hypothesis, to counteract such an unfavourable, uncontrolled cell development? Obviously, it would be a stricter control of restraints, a limitation of energy access from outside. It would be a therapy of controlled fasting! Does it make sense? Fact is that since antiquity natural philosophers and doctors (Pythagoras, Hippokrates, Asklepiades, Galen, Benedict of Nursia, Kneipp, and many modern experienced physicians) recommend fasting against certain severe diseases. Fasting is, of course, also known to be a relevant religious and health related element in other cultures worldwide. There may be a deeper meaning behind and a hint at the possible fundamental origin of uncontrolled growth, 
when constraints become weak or break down. In this case not a genetic modification causing uncontrolled growth, but a genetic failure of not limiting growth should be in the focus of interest.

Corpulence and obesity are other situations, which may be related to a fundamental tendency of self-organized systems towards increasing energy turnover and entropy production. It is now often explained as a behavioural relict from stone age, where food was scarce and not always available. This makes sense, but it could also be related to the discussed inherent and fundamental disposition of self-organized life towards increasing energy turnover, which surfaces under certain genetic, social and environmental conditions. What could speak for such an interpretation is the observation that populations originating from very different cultural backgrounds and geographic (arctic, tropic) environments in the world seem to become equally exposed to such problems, when abundant food is available.

\section{Discussion}

Accepting a fundamental time arrow as a paradigm change for physics and natural science means understanding that a (dynamic) energy is driving time in form of a flow of action (which is proposed to be the ultimate manifestation of time in nature) (Tributsch, 2015, 2016a). Energy turnover (and not higher probability) is the cause for observed changes and the passage of time. This, as shown here, is not in contradiction with the presently accepted probabilistic time concept towards higher probability and increasing entropy. It includes it as a special case, since entropy increase $\Delta S$ in a probabilistic, statistical ensemble means increase of not any more available (entropic) energy (T $\Delta \mathrm{S})$. It must arise from available energy. The fundamental time arrow, however, which could be derived from the important principle of least action (Tributsch, 2016a), opens the possibility to enable and understand fast responding feedback processes as required for self-organization in molecular systems, in evolution and development of human consciousness and spirit. It also allows deriving and understanding the second law of thermodynamics, which conventional physics cannot do. Most important, it also yields the entropy law governing self-organized systems such as life, ecosystems or galactic phenomena: maximum entropy production (energy turnover) within the restraints of the system will be approached. Genetic mechanisms, themselves proposed to be self-organized, evolved dealing with, controlling and safeguarding such a fundamental aimed property of self-organized biological systems.

The paradigm change towards a fundamental time arrow entrains very different consequences for understanding irreversible processes and evolution of life, as Figure 3 visualizes. The entropy law, which aims at maximum entropy (energy) turnover (within the restraints of the system), is fundamental and gives life the direction, the determination, the hunger for energy. It must be considered superposed to and interacting with the DNA controlled, genetic mechanisms of 


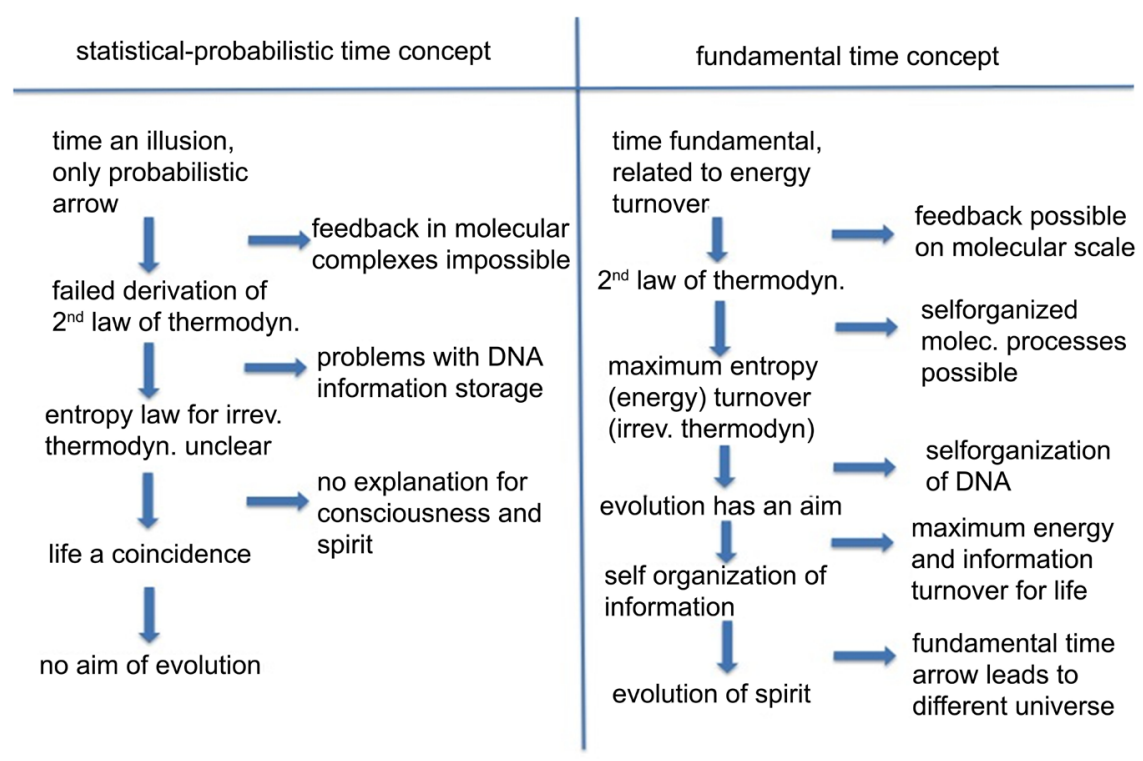

Figure 3. Scheme comparing implications of statistical, probabilistic time (left) and of fundamental (energy driven) time arrow (right).

biological evolution. When considered to be self-organized, chemical DNA information will aim at creating new self-similar information which is the basis of new life and continuation in evolution.

The here presented approach opens a new dimension for understanding and argumentation. According to the explained concept, statistical mutations function and develop superposed to the law of maximum energy (entropy) turnover of self-organized systems, which self-organized genetic mechanisms are safeguarding. Mutations, which occur in presence of abundant energy, have a higher probability to succeed and resulting energetic changes in the self-organized living systems can induce altered structural patterns or they may change the constraints. Such a superposed activity is expected to shape the progress of evolution in a more pronounced and better focussed way than arbitrary micro-mutations alone. Before all it is immediately clear why there is a characteristic orientation in life: it is the ability and drive to harvest additional energy for the sustainment of self-organized activities. It is contained in food, in solar or environmental energy. Here the claimed fundamental property of self-organized systems towards maximising entropy (energy) turnover becomes evident.

The genetic mechanism, through natural selection, is involved in perpetuating and safeguarding life and automatically functions within this law of maximum entropy (energy) turnover because outside a characteristic parameter space life does not exist as a self-organized system. Genetic mechanisms and genetic information evolved towards avoiding boundaries, where excessive energy turnover could lead to a breakdown of self-organization. Evolutionary pathways and natural selection thus guide life towards increasing entropy (energy) turnover (provided the external and internal restraints allow it). Otherwise damaging and destructive processes of uncontrolled growth (as mentioned above) could easily 
take over.

When accepting a fundamental time arrow and its consequences time is not any more an illusion. It is the track which energy turnover is carving into our environment and into our brain. Biological evolution as well visualizes this track. From the flow of action, associated with energy turnover, clock time can be extracted via calibration. This is actually the process proceeding in clocks.

Subject to such a fundamental time arrow, the universe has to be understood quite differently and evolution in general, geological and biological evolution, has an aim (Tributsch, 2015; 2016b; 2018a). This aim of evolution is maximizing entropy and energy turnover respectively, within given constraints. It includes, in biological evolution, the shaping property of the genetic code (with the here emphasized self-organized function) and natural selection. The essential difference to the conventional understanding of evolution is that up to now no fundamental property, in terms of an entropy law, has been attributed to self-organized inorganic and living system themselves. Life was just considered to be a given, neutral phenomenon, exposed to arbitrary statistical mutations and natural selection.

In contrast, according to the here proposed considerations natural selection is not a process of chance only, but, with the properties of self-organized systems, includes a clearly deterministic element. It concerns the turnover and acquisition of energy. Natural selection acts on systems that aim at building up order by maximising entropy production (energy turnover). Natural selection is a shaping force, while the acquired energy flows towards maximum energy turnover are the orienting and driving forces. They sustain life and define the orientation, because they favour mutations which support energy flows. And additional energy flows can induce new self-organization patterns, since they can push the systems further away from equilibrium and may change the restraints. During biogenic and anthropogenic evolution these energy thrusts included the discovery of photosynthesis, the availability of oxygen in the atmosphere, the discovery of fire by humans, of agriculture, and the use of hydrocarbons.

Is such a statement of an aim in evolution, which is denied by conventional evolution research, standing alone? It is not. Such a conclusion is also consistent with the result of another study, based on different arguments, claiming a "direction of evolution" (Stewart, 2014), which recognizes an "increasing global diversification and integration". It is explained in terms of an observed optimization of exploitation of global resources. This appears to be basically equivalent to the here proposed aim of maximising entropy production (energy turnover). As long as additional energy can be increasingly harvested and self-organization is sustained, evolution follows an aim. This emphasizes the role of energy in shaping evolution, which has also been highlighted in another study (Niele, 2005). Open self-organized systems tend to favour a growing energy input und through-flux. Since increasing energy turnover is paralleled by an increasing shift from equilibrium and thus an increasing ability for generating order this 
explains evolution towards higher perfection, but also towards an increasing rate of implemented mutations (as identified by Hawks et al., 2007). It also could explain why the biggest mammals and plants, blue whale and sequoia, and the species with the highest specific energy consumption, humans, are living today.

Information (related to energy) can also self-organize subject to a fundamental time arrow, because fast feedback processes can function in a highly ordered environment, and are able to create a higher hierarchy of information handling above mere computation. This is recognized as the phenomenon of consciousness and spirit, which thus can be explained on purely materialistic basis (Tributsch, 2016b; 2015). It is subject to similar boundary conditions as evolution of material life. It may now be easier to understand the reason why the human brain and consciousness had such a fast start (one to two million years), compared to more than 3.5 billion years of evolution of life. They significantly accelerated energy (entropy) turnover and thus gave access to new successful structural and spiritual patterns.

All these results and conclusions appear to be compatible with experience and observations. There is evolution towards higher complexity (compare however deviating opinions, Gould, 1996; Wilkins, 1997), mankind moved towards increased energy turnover and there is human consciousness and spirit. This in turn inevitably requires that a fundamental time arrow is accepted for our world and the universe. A probabilistic statistical time arrow and an energy without interest to do work cannot give reasonable access to the complex world of irreversible thermodynamics including the phenomena of life. Subject to a fundamental time arrow and its consequences complex properties of evolution become more easily understandable.

The energy hunger of living species arose from their property as self-organized systems. Also their ability to form symbiotic unions, which significantly increased their energy input, has the same origin. However, many open questions remain. For example, the mutual interaction between the energy hunger of self-organized living systems and the (proposed) self-organized genetic code could become a major challenge for future evolution research. An interaction and synchronization between self-organized mechanisms is possible and bears the potential for new insight into evolution towards higher complexity.

All these considerations on an aim in evolution, resulting from the superposition of genetic mechanisms to a trend of maximizing entropy turnover, are falsifiable (procedure according to science philosopher Popper, 1979). They are wrong, if it can be shown that self-organized systems (like a hurricane, the light utilizing Belousov-Zhabotinsky reaction, or life) do not allow maximum entropy (energy) turnover, within their restraints. They are also falsified, if it can be shown that life cannot be treated as a self-organized system. They can also be falsified by demonstrating that a natural process can entirely be reversed without additional changes, demonstrating time neutrality and absence of a fundamental energy driven time arrow. 
Maximum entropy production (energy turnover) as a fate for human civilization is, unfortunately, a quite pessimistic prospect due to unavoidable environmental consequences. If developments remain unchecked (and adequate spirit is not involved in counteracting) humans will go on eliminating restraints and will eventually destroy their environment by accumulating excessive entropy from energy conversion (atmospheric $\mathrm{CO}_{2}, \mathrm{CH}_{4}$, radioactive contamination, particulate air pollution, heat pollution, pollution with plastic materials, pesticides, pharmaceutical pollution in water). Of course, human spirit and farsighted governments have already recognized that on an empirical basis.

The result that biological evolution fundamentally aims at maximum entropy production (energy turnover) is a quite serious and disturbing claim. However, it follows in a straightforward way from a fundamental time arrow and the identification of the entropy law, which determines self-organized systems. This means, such a development is unavoidable, if intelligence and spiritual determination does not counteract by controlling the restraints. It is therefore necessary to discuss this claim in depth, considering that, at present, the established opinion is that evolution has no aim (and that its manifestations are only consequences of complex past and present environmental conditions).

However, evolution of spirit is a parallel consequence of a maximising entropy production (energy turnover), since it becomes only possible when high energy turnover is achieved in brain systems. As a more optimistic alternative, this evolution of spirit may therefore be considered the ultimate aim of evolution. According to the arguments discussed here spirit could only evolve because evolution aims at maximising entropy production (energy turnover) and actually succeeded in providing the necessary high energy through-flux in the brain. Assuming evolution of spirit as the real aim of evolution is much more attractive not only because of psychological reasons, but also because it is a more fascinating and creative idea. In addition, this exactly suggests how humans could finally avoid environmental destruction as a consequence of maximised energy turnover. A creative human spirit could ultimately dominate and control environmental destruction by limiting restraints for maximum entropy production. The best option, according to the author's opinion, would be to reintegrate human energy technology, in a bio-mimetic effort and on a high technical level, again into nature's energy strategy (Tributsch, 2008b \& 2012). This is reasonable, because the natural energy strategy also evolved the climate, which itself is a self-organized system, and thus also developed towards maximum entropy turnover. And life itself synchronized with (adapted to) this climate. The fact, by the way, that living nature itself has shaped atmospheric conditions and climate towards allowing higher energy turnover (e.g. with oxygen for living species), already supports the claim, that life and the environment are aiming at maximum entropy production (energy turnover). They are shaped that way and they tend to absorb available additional energy as long as their restraints permit it.

The integration of human energy technology back into nature's strategy has 
been discussed in detail (Tributsch, 2008b \& 2012) and has the advantage that, once reached, maybe within one century, a reasonably favourable climate on earth could be sustained even with a high level of human energy consumption. Such a bio-mimetic strategy would allow an adequate balance between the encountered drive for maximum energy turnover and intelligent restrictions in human activities, enabling a narrowing of restraints.

The proposal that evolution of spirit is the ultimate aim of biological evolution would drastically change our views on the fate of the human race. Since only purely materialistic considerations have led to this surprising concept they should be applicable all over the universe. As a consequence, higher life and spirit, with the inherent drive to increase energy turnover, should be frequent in the universe and there could be spiritually very advanced civilizations spread over the galaxies, that have learned to deal with entropy pollution. The reason is that also (energy converting) information turnover and spirit have an aim: maximum information turnover within the constraints of the system. It would be such civilizations, which succeeded in properly handling high rates of energy and information turnover, while conserving an environment adequate for survival that would reach a very high level of spirit. The claimed trend of maximising information turnover (within the restraints of the system) may, by the way, already be recognized within our still young information age, and in the behaviour of the young generation. In addition, it can be observed in the increasing rate of digitalization and automatization of industry and traffic, as well as of urban structures.

The main conclusion from this investigation that evolution based on an energy driven time arrow has an aim, maximum entropy (energy) turnover and evolution of spirit respectively, is a teleological statement, a statement in function of its goal. It is well known that modern science and philosophy are very critical with respect to such statements. In biology, teleological statements are typically avoided. However, feedback controlled processes, which living systems represent, necessarily suggest intrinsic aims, irrespective of human opinions. This has already been recognized by Rosenblueth, Wiener and Bigelow (1943), who defined teleology as "feedback controlled purpose". Cybernetics, the study of regulatory feedback, was seen by Norbert Wiener to deal with teleological mechanisms. In addition, philosopher Nagel (2012) even invokes "a natural teleological law" to explain life and consciousness. Such a law (maximum entropy production) has been derived and explained in this paper. Critics of teleological reasoning will have to disprove it. Since it is based on feedback driven mechanisms, teleological response is an automatic consequence.

Then there is the philosophical question why there should be an aim in the universe towards development of spirit. While the arguments advanced here are purely materialistic it is also possible to imagine a spiritual reason behind: a superior power (natural or a deity) that favours evolution of intelligent beings according to its image. This gives ample room for philosophical or religious considerations. The gap in understanding, between materialistic, intelligent design, 
and religious interpretations of evolution would be significantly narrowed. And spirit as aim of evolution could, in a sensitive way, unbalance argumentations in ongoing disputes on religion and society (Dawkins, 2006; McGrath, 2007). Before all, humans will feel better, knowing that with their evolving spirit they are privileged and can take active part in evolution. They may feel more responsible in safeguarding their precious environment, which enables and supports such a privilege. Such considerations may ultimately lead to a new philosophy (or religion), helping humans to find their place in the universe and to tackle problems in an increasingly complex, futuristic environment.

All conclusions discussed here are based on a paradigm change: natural phenomena are not time-neutral, as taken for granted today, but fundamentally time oriented and energy driven. Science philosopher and science critic Feyerabend (1986) emphasized the role of induction and counter induction, besides of the methodical collection of information, and of rhetorical efforts for the evolution and stabilization of a recognized world model. Assuming a time neutral fundamental nature, which is the basis of our present established scientific world model, means implementing counter induction, since it is a hypothesis (from the time of Newton), which contradicts reality. There is no experimental proof for it, while irreversibility and self-organization in nature is omnipresent. The author (Tributsch, 2015) argues and shows that counterintuitive paradoxes in quantum physics and cosmology and the increasing trend towards irrational and abstract mathematical hypotheses (effects without causes, multiple dimensions, time travel, parallel worlds, energy and particles popping out from nothing) are constructions required to support such an incorrect basic assumption within expanding and more detailed theories. If a fundamental energy driven time arrow is assumed, paradoxes are avoided, rational explanations become possible, and a quite different universe is recognizable. On the other hand, practical knowledge based on time-neutral assumptions can still be handled as limiting cases and remains understandable and applicable. Extrapolation from time neutrality towards irreversibility is, on the other hand, impossible. A paradigm change concerning the concept of time is therefore unavoidable.

Basing on an energy driven time arrow, which facilitates feedback processes this paper explained the energy hunger of living organisms and the human drive towards increasing energy (entropy) turnover as phenomena of self-organization. Along the same line self-organization of genetic and neuron based information explains self-similar information handling in evolution and self-organization of brain function to support consciousness and spirit. These phenomena really exist and could not be adequately classified and explained before. This in turn supports a paradigm change towards an energy driven time arrow, but simultaneously would reshape concepts of evolution. Profound discussions are needed.

\section{Outlook}

There is another point, which is relevant when dealing with an aim in evolution 
and with information and spirit in living beings within an evolving universe. The presently favoured physical concept of the universe is an entirely pessimistic one. The universe is hostile and unfriendly. It arose from a catastrophic Big Bang explosion, is involved in a mysterious expansion of "empty" space, accelerates galaxies to near light velocity and will end in a cold nothingness. Life is just a coincidence, a statistical play of parameters, mutations and selection, which developed due to a very narrow chance constellation of conditions, and evolved without aim. Even more puzzling: everything in this highly dynamic scenario is explained on the basis of time-neutral elementary particles and laws describing them, with a time only following a probabilistic path.

The concept of a fundamental energy driven time arrow, which is proposed here as a paradigm change, draws a very different situation, a nature, which is fundamentally irreversible. When re-evaluating the meaning of quantum states and the particle-wave duality (which lose their time-neutral status), it turned out that what we have learned to be "gravitation" is facilitating the reconstruction of a particle from a wave and is nothing else than information (on matter) (Tributsch, 2016a, 2016b, 2018b). Gravitation (identified as information) is however also known to be controlling the dynamics of the universe. It makes consequently sense to conclude that information is a really dominating factor in the universe (that natural laws allow information handling on an impressive level is already demonstrated by our fast growing information technology). Information may have created a starting universe with low entropy and may ultimately recreate it from a wornout high entropy one (Tributsch, 2015; 2016b). Biological evolution of spirit is a fascinating process but may only be one aspect and well fitting into a universe, which would be largely controlled by information. The function of the universe could be much more intelligent than presently assumed and the awakening human spirit may just be one manifestation of its creative ability. A fundamental time arrow can help us explore this still hidden character of our universe and better understand the role of biological evolution within. This may also help answer a question, posed by Nagel (2012), as to the nature of a universe, which can evolve beings who try to understand it. Such a universe may be considered basically friendly. It allows and encourages spirit and civilizations to grow.

\section{Conflict of Interest}

The author declares to have no conflict of interests.

\section{References}

Blankenship, R. E. (2010). Early Evolution of Photosynthesis. Future Perspectives in Plant Biology. http://www.plantphysiol.org/content/154/2/434.full

Chen, Z., Miller, E., \& Dinh, H. N. Editors. (2013). Photoelectrochemical Water Splitting. Cham: Springer Briefs in Energy.

Dawkins, R. (2006). The God Delusion. Boston: Houghton Mifflin.

Dharma-Wardana, C. (2013). A Physicist's View of Matter and Mind. Singapore: World 
Scientific.

Feyerabend, P. (1986). Wider den Methodenzwang. Frankfurt: Suhrkamp.

Fujishima, A., Hashimoto, K., \& Watanabe, T. (1999). TiO ${ }_{2}$ Photocatalysis: Fundamentals and Applications. Tokyo: BKC.

Futuyma, J. D., \& Kirkpatrick, M. (2017). Evolution (4th Ed.). Sinauer, Sunderland, MA: Sinauer Associates.

Gould, S. J. (1996). Life's Grandeur: The Spread of Excellence from Plato to Darwin, Random House. https://doi.org/10.4159/harvard.9780674063396

Hawks, J., Wang, T. E., Cochran, G. M., Harpending, H. C., \& Moyzis, R. K. (2007). Recent Acceleration of Human Adaptive Evolution. PNAS, 104, 20753-20758. https://doi.org/10.1073/pnas.0707650104

Hesse, J., \& Gross, T. (2014). Self-Organized Criticallity as a Fundamental Property of neural Systems. Frontiers in Systems Neuroscience. https://doi.org/10.3389/fnsys.2014.00166

Izhikevich, E. M. (2007). Dynamical Systems in Neuroscience. Cambridge, Massachusetts: The MIT Press.

Jung, C. G. (1958-1981) Gesammelte Werke (Collected Work). Freiburg: Walter.

Kolling, D. R. J., Cox, N., Ananyev, G. M., Pace, R. J., \& Dimukes, G. C. (2012). What Are the Oxidation States of Manganese Required to Catalyze Photosynthetic Water Oxidation. Biophysical Journal, 103, 313-322. https://doi.org/10.1016/j.bpj.2012.05.031

Lewerenz, H.-J., \& Peter, L. Eds. (2013). Photoelectrochemical Water Splitting: Materials, Processes and Architectures. Royal Society of Chemistry Book. http://pubs.rsc.org/en/content/ebook/978-1-84973-647-3\#!divbookcontent

Liu, Y., Liu, C., \& Wang, D. (2011). Understanding Atmospheric Behavior in Terms of Entropy: A Review of Applications of the Second Law of Thermodynamics to Meteorology. Entropy, 13, 211-240.

Lynch, M. (2007). The Origins of Genome Architecture. Sunderland, MA: Sinauer Associates Inc.

McElheny, V. K. (2012). Drawing the Map of Life: Inside the Human Genome Project. London: Hachette.

McGrath, A. (2007). The Dawkin Delusion: Atheist Fundamentalism and the Denial of the Divine.

Mukhopadhyay, S., Mandal, S. K., Bhaduri, S., \& Armstrong, W. H. (2004). Manganese Clusters with Relevance to Photosystem II. Chemical Reviews, 104, 3981-4026. http://nptel.ac.in/courses/102104043/AM08.pdf

Mullen, L. (2004). Clues to Life in the Mines of Murgul. Astrobiology Magazine. http://www.astrobio.net/news-exclusive/clues-to-life-in-the-mines-of-murgul/

Nagel, T. (1974). What Is It Like to Be a Bat. Philosophical Review, 83, 435-450. https://doi.org/10.2307/2183914

Nagel, T. (2012). Mind and Cosmos, Why the Materialist Neo-Darwinian Conception of Nature Is Almost Certainly False. Oxford: Oxford University Press. https://doi.org/10.1093/acprof:oso/9780199919758.001.0001

Niele, F. (2005). Energy: Engine of Evolution. New York, NY: Elsevier.

Novak, M. A. (2006). Evolutionary Dynamics. Cambridge, MA: Harvard University Press.

Okasha, O. (2006). Evolution and the Levels of Selection. Oxford: Clarendon Press. https://doi.org/10.1093/acprof:oso/9780199267972.001.0001 
Paltridge, G. W. (1979). Climate and Thermodynamic Systems at Maximum Dissipation. Nature, 279, 630-631. https://doi.org/10.1038/279630a0

Pohlmann, L., \& Tributsch, H. (1993). A Hypothesis Explaining the Activated Complex in Primary Photosynthetic Electron Transfer as a Dissipative Structure. The Journal of Physical Chemistry, 97, 11318-11323. https://doi.org/10.1021/j100145a033

Popper, K. R. (1979). Die beiden Grundprobleme der Erkenntnistheorie. In J. C. B. Mohr, \& P. Siebeck (Eds.), Herausgeber: Troels Eggers Hansen (pp. 426-427). Tübingen.

Rhee, K. H., Morris, E. P., Zheleva, D., Hankamer, B., Kühlbrandt, W., \& Barber, J. (1997). Two-Dimensional Structure of Plant Photosystem II at 8-A Resolution. Nature, 389, 522-526.

Rosenblueth, A., Wiener, N., \& Bigelow, J. (1943). Behaviour, Purpose and Teleology. Philosophy of Science, 10, 18-24.

Ross, J. (2008). Thermodynamics and Fluctuations Far from Equilibrium (12.5: Invalidity of the Principle of Maximum Entropy Production) (p. 119). Berlin: Springer. https://doi.org/10.1007/978-3-540-74555-6

Ruse, M. (1996). Monad to Man: The Concept of Progress in Evolutionary Biology. Cambridge, MA: Harvard University Press.

Stewart, J. E. (2014). The Direction of Evolution: The Rise of Cooperative Organization. Biosystems, 123, 27-36. https://doi.org/10.1016/j.biosystems.2014.05.006

Swenson, R. (1997). Autocatakinetics, Evolution, and the Law of Maximum Entropy Production: A Principled Foundation towards the Study of Human Ecology. Advances in Human Ecology, 6, 1-47. http://rodswenson.com/humaneco.pdf

Tributsch, H., Fiechter, S., Jokisch, D., Rojas, J., \& Ellmer, K. (2003). Photoelectrochemical Power, Chemical Energy and Catalyic Activity for Organic Evolution on Natural Pyrite Interfaces. Origin of Life and Evolution of the Biosphere, 33, 129-162. https://doi.org/10.1023/A:1024613216477

Tributsch, H. (2008a). Photovoltaic Hydrogen Generation. International Journal of Hydrogen Energy, 33, 5911-5930. https://doi.org/10.1016/j.ijhydene.2008.08.017

Tributsch, H. (2008b). Erde, Wohin Gehst Du? Solare Bionik-Strategie: Energie-Zukunft nach dem Vorbild der Natur (346 p.). Aachen: Shaker Media.

Tributsch, H. (2012). Energy Bionics: The Bio-Analogue Strategy for a Sustainable Energy Future. In N. Z. Muradov, \& T. N. Veziroglu (Eds.), Carbon-Neutral Fuels and Energy Carriers. Boca Raton: CRC Press, Taylor \& Francis.

Tributsch, H. (2015). Irrationality in Nature or in Science? Probing a Rational Energy and Mind World. Scotts Valley, CA: Create Space.

Tributsch, H. (2016a). On the Fundamental Meaning of the Principle of Least Action and Consequences for a "Dynamic" Quantum Physics. Journal of Modern Physics, 7, 365-374. http://www.scirp.org/journal/jmp https://doi.org/10.4236/jmp.2016.74037

Tributsch, H. (2016b). A Fundamentally Irreversible World as an Opportunity towards a Consistent Understanding of Quantum and Cosmological Contexts. Journal of Modern Physics, 7, 1455-1482. http://www.scirp.org/journal/jmp https://doi.org/10.4236/jmp.2016.712133

Tributsch, H. (2018a). Elementary Particles Subject to an Energy Driven Fundamental Time Arrow. Journal of Modern Physics, 9, 1361-1380. https://doi.org/10.4236/jmp.2018.97082

Tributsch, H. (2018b). The Challenge of Water Splitting in View of Photosynthetic Reality and of Research Trends; Energy and Environment Series No. 20, Advances in Pho- 
toelectrochemical Water Splitting: Theory, Experiment and Systems Analysis. In S. David Tilley, S. Lany, \& R. van de Krol (Eds.), The Royal Society of Chemistry (RSC).

Wächtershäuser, G. (2000). Perspectives. Origin of Life: Life as We Don't Know It. Science, 298, 1307-1308.

Watanabe, T., Kobayashi, M., \& Sagara, T. (1990). Electrochemical and Photochemical Interpretation of Oxygen Evolution Process. In M. Baltscheffsky (Ed.), Proceedings of the 8th International Conference on Photosynthesis Stockholm (pp. 885-888). Dordrecht, The Netherlands: Kluwer.

Wilkins, J. S. (1997). Evolution and Philosophy. Is There Progress and Direction in Evolution? http://www.talkorigins.org/faqs/evolphil/teleology.html 